\title{
Parasite Infiltration and Apoptosis in Spleen upon Toxoplasma gondii Infection
}

\author{
Su-Hwa Lee', Ki-Back Chu', Fu-Shi Quan²,3,* \\ ${ }^{1}$ Department of Biomedical Science, Graduate School, Kyung Hee University, Seoul 02447, Korea; '2Department of Medical Zoology, Kyung Hee \\ University School of Medicine, Seoul 02447, Korea; ${ }^{3}$ Medical Research Center for Bioreaction to Reactive Oxygen Species and Biomedical Science \\ Institute, School of Medicine, Graduate School, Kyung Hee University, Seoul 02447, Korea
}

\begin{abstract}
Toxoplasma gondii infection induces parasite infiltration and apoptosis in the spleen. However, dose-dependent parasite infiltration, apoptosis, body weight alternations and survival in mice remain largely unknown. In this study, mice were intraperitoneally infected with 10,30 or 100 tachyzoites of $T$. gondii, respectively. Parasite infiltration and apoptosis in the spleen were analyzed on days 3, 7, and 9 post-infection by immunohistochemistry and flow cytometry. Significantly higher levels of T. gondii infiltration and apoptosis in the spleen were found in 30 and 100 tachyzoites infected mice compared to 10 tachyzoites infected mice on days 7 and 9 post-infection. Although 30 and 100 tachyzoites infected mice showed significant body weight loss compared to 10 tachyzoites infected mice, all of the 100, 30, and 10 tachyzoites infected mice died by days 12, 15, and 17, each respectively. Interestingly, T. gondii infiltration in 10 tachyzoites infected mice were limited to capsule area of the spleen on day 9 post-infection. Several areas of parasite infiltrations were found in the 30 tachyzoites infected mice, where noticeable levels of splenic capsule de-adhesion occurred. These results indicated that parasite infiltration and apoptosis in the spleen, as well as body weight loss (survival) are closely correlated with infection dosage. The level of $T$. gondii infiltration and apoptosis in the spleen and splenic de-adhesion were dependent on the parasite dose.
\end{abstract}

Key words: Toxoplasma gondii, infiltration, apoptosis

Toxoplasma gondii, the causative agent of toxoplasmosis, is an ubiquitous protozoan parasite that can infect wide range of host species including humans [1]. It is estimated that around $30-50 \%$ of the world's population have been infected with $T$. gondii [2]. T. gondii infections are typically asymptomatic in humans, however, these can be serious and occasionally fatal in the case of immunocompromised patients, fetus and congenitally infected neonates $[3,4]$. T. gondii can exist in three different forms, which are largely influenced by the host it inhabits. Within its definitive host (Felidae), T. gondii are found as oocysts whereas tachyzoite and bradyzoite are the predominant forms in other intermediate hosts. Oocysts in the feces of definitive host can be ingested by other intermediate hosts, which results in infection. Mice as intermediate hosts are extensively used for pathogenesis and vaccine study of toxoplas-

- Received 4 June 2019, revised 16 August 2019, accepted 25 August 2019.

*Corresponding author (fsquan@khu.ac.kr)

(c) 2019. Korean Society for Parasitology and Tropical Medicine

This is an Open Access article distributed under the terms of the Creative Commons Attribution Non-Commercial License (http://creativecommons.org/licenses/by-nc/4.0) which permits unrestricted non-commercial use, distribution, and reproduction in any medium, provided the original work is properly cited. mosis, in which high numbers of tachyzoites of T. gondii (RH) $\left(10^{3}, 10^{4}, 10^{5}\right)$ have been used [5-12]. T. gondii (RH) is highly virulent and its infection in mice causes death [13]. Mice infected with $10^{5}$ tachyzoites show no $\mathrm{CD}^{+} \mathrm{T}$ and germinal center B cell responses from the spleen at day 16 post-infection, since these immune cells are largely damaged [12]. Low vaccine efficacies were reported upon challenge infection with $T$. gondii $(\mathrm{RH})$ at high dosage $\left(10^{3}, 10^{4}, 10^{5}\right)$, in which mice died at very early stage of infection and immune responses elicited by challenge infection cannot be detected [5-11]. Thus, finding correct infection dosage for challenge infection to evaluate vaccine efficacy and immune responses is extremely important.

Importantly, there are controversial reports on the possibility of infection of T. gondii tachyzoites in the spleen. It has been reported that $T$. gondii cannot be found in the spleen, whereas parasites can be detected in the lung, liver and brain upon tachyzoite infection [14]. In contrast, others have reported that tachyzoites of $T$. gondii can infect the spleen $[15,16]$. Thus, more studies are needed to clarify the controversy underlying splenic infectivity by T. gondii tachyzoites. Apoptosis is programmed cell death, which mediates the removal of pathogens 
[17]. Recently, apoptotic response has been reported to be elicited in the spleen upon $T$. gondii tachyzoite infection in mice $[10,11]$. Thus, we believe that apoptotic responses occurred in the spleen after T. gondii infiltration into spleen. In this study, we investigated parasite infiltration and apoptosis induced by various dosage of tachyzoites of T. gondii in the spleen. We focused on clarifying the low dose of tachyzoite infection-induced pathogenesis. We found that much lower dose of T. gondii tachyzoite can enter the spleen and cause apoptosis.

Specific-pathogen-free female BALB/c mice (7 weeks old) were obtained from NARA Biotech (Seoul, Korea). All animal experiments and husbandry involved in the present study were conducted under the guidelines of Kyung Hee University IACUC (permit number: KHUASP [SE]-16-012). T. gondii RH strain was maintained by serial intraperitoneal passage in mice as described previously $[12,18,19]$. T. gondii ME49 strain was maintained by oral passage in mice to produce polyclonal anti-T. gondii antibodies as described previously [12]. Horseradish peroxidase (HRP)-conjugated goat anti-mouse immunoglobulin IgG was purchased from Southern Biotech (Birmingham, Alabama, USA). BALB/c mice were randomly divided into 4 groups ( $\mathrm{n}=24$ per group): naïve control group, 10 tachyzoites infected group (10), 30 tachyzoites infected group (30) and 100 tachyzoites infected group (100). Mice were infected intraperitoneally (IP) with 10, 30 or 100 tachyzoites of $T$. gondii RH strain and sacrificed ( 6 from each group) on days 3 , 7 , and 9 post-infections to collect spleen samples. The remaining 6 mice were observed daily to monitor changes in body weight and survival. Mice that displayed over $20 \%$ loss in body weight were considered dead and humanely euthanized. To determine apoptotic response in splenocytes, Annexin V and propidium iodide (PI) staining were performed using BD
A

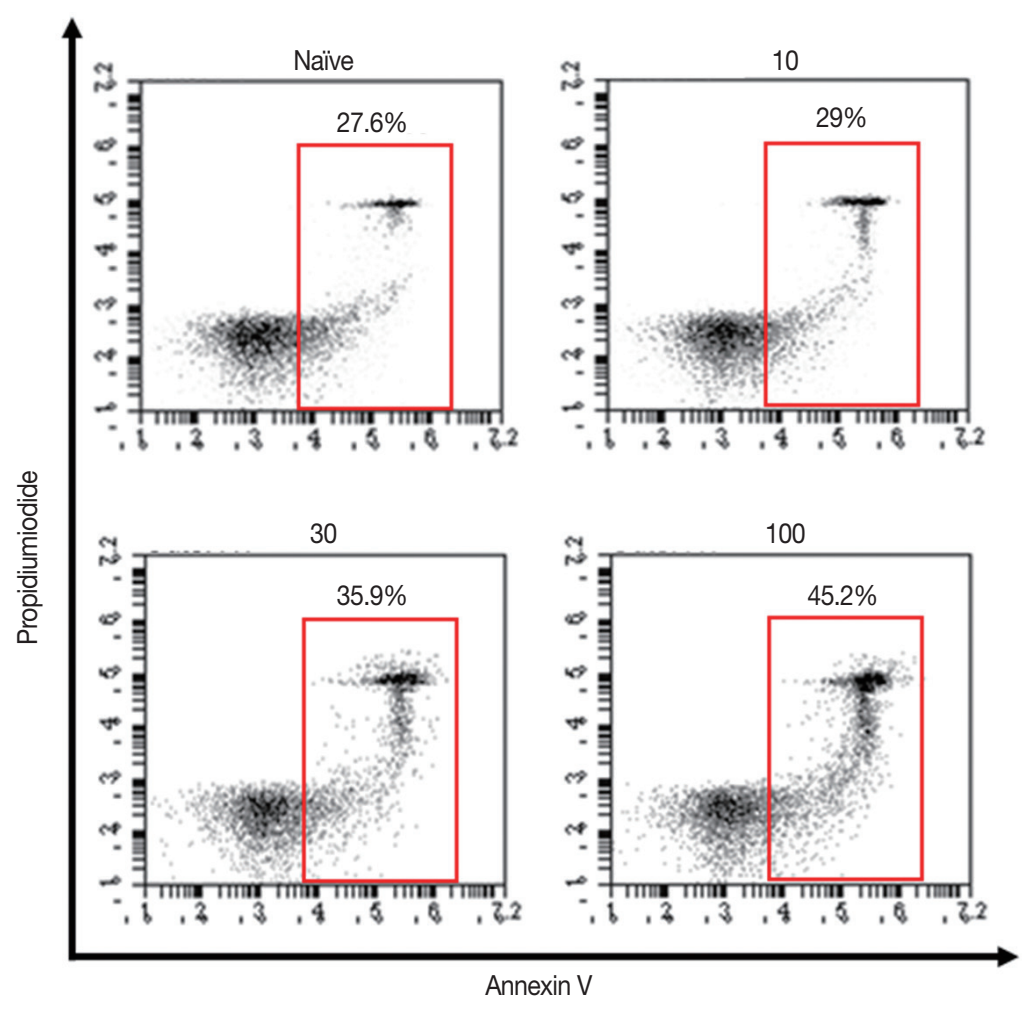

B

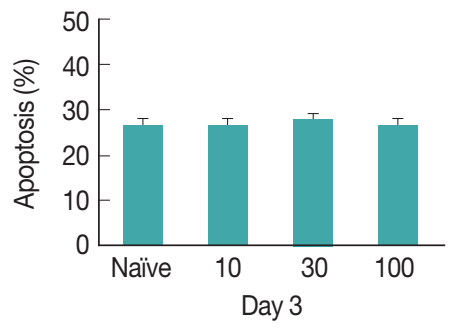

C

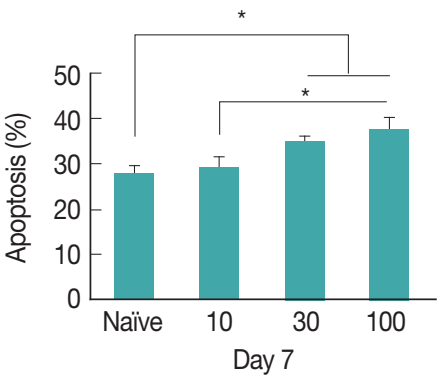

D

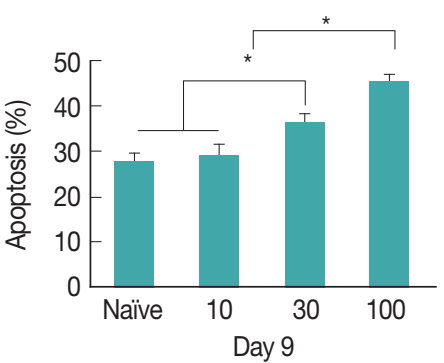

Fig. 1. Apoptotic responses upon T. gondii tachyzoite infection. (A) Flow cytometry plots showing the gating strategy to identify apoptotic responses in the spleen (10, 10 tachyzoites infected mice; 30, 30 tachyzoites infected mice; 100, 100 tachyzoites infected mice). (B-D) The apoptotic response was determined by flow cytometry at days 3, 7, and 9 after $T$. gondii infection. Significantly higher levels of apoptotic effects were found from both 30 and 100 mice on days 7 and day 9, each respectively compared to naïve control mice on day 7 . 
Apoptosis Detection Kit I (BD Biosciences, San Jose, California, USA). Splenocytes were collected on days 3,7 , and 9 postinfections and $1 \times 10^{5}$ cells were stained with $5 \mu \mathrm{l}$ Annexin VFITC and PI at room temperature for $15 \mathrm{~min}$ in the dark. The percentages of apoptotic response were determined using $\mathrm{BD}$ Accuri C6 Flow Cytometer (BD Biosciences) and analyzed with C6 Analysis Software (BD Biosciences). Mice spleen were collected at day 9 post-infection and fixed in 10\% formalin. The formalin-fixed paraffin-embedded (FFPE) spleens were prepared and cut into micro-sections as previously indicated [20]. Immunostaining procedures were performed after removing paraffin with xylene and rehydrating with decreasing concentrations of ethanol in distilled water. After antigen retrieval in $10 \mathrm{mM}$ citrate buffer ( $\mathrm{pH}$ 6.0), endogenous peroxidase activity blockage was obtained by $3 \%$ peroxide hydrogen solution treatment. The FFPE sections were blocked with blocking solution (1\% BSA in $0.1 \mathrm{M} \mathrm{PBS})$ and stained with a mouse polyclonal anti-T. gondii ME49 antibodies $(1: 2,000)$ diluted with $1 \%$ bovine serum albumin (BSA) in tris-buffered saline with tween 20 (TBST) for $1 \mathrm{hr}$ at RT. An amplification signal was obtained by horseradish peroxidase (HRP)-conjugated goat anti-mouse immunoglobulin IgG, in 30 min incubation step at RT. The reaction was visualized by using $0.2 \%$ of 3,3-diaminobenzidine tetrahydrochloride (DAB). Finally, the sections were counterstained with hematoxylin for back- ground stain after DAB stain. Statistical analyses were performed using PC-SAS 9.3 software (SAS Institute, Cary, North Carolina, USA). Data sets were expressed as mean \pm SEM and compared using 1-way ANOVA with Duncan's post hoc analysis. A $P$-value $<0.01$ was considered statistically significant.

To determine apoptotic response induced by T. gondii infection, splenocytes were collected from mice and analyzed by FACS (Fig. 1A). Mice infected with T. gondii showed various apoptotic effects by infectious doses at different time points. Mice showed no apoptotic responses at early stage of infection at day 3 (Fig. 1B). However, mice infected with 100 tachyzoites (100) showed $37.4 \%$ and $45.2 \%$ of apoptotic responses on days 7 and 9, respectively (Fig. 1C, D). Thirty tachyzoites infected mice (30) showed $34.8 \%$ and $35.9 \%$ of apoptotic responses on days 7 and 9, respectively (Fig. 1C, D). On day 9 post-infection, 100 tachyzoites infected (100) mice showed significantly higher level of apoptotic effects compared to 30 tachyzoites infected ( $45.2 \%$ vs $35.9 \%,{ }^{*} P<0.01$ ). These results indicated that apoptosis in the spleen occurs at day 7 post-infection with a high dosage of infection, whereas negligible apoptotic response from low dose of infection (10) were observed by day 9 post-infection.

Splenic T. gondii infiltration was determined by immunohistochemistry. As shown in Fig. 2, T. gondii infiltration was found in all mice infected with 10, 30 or 100 tachyzoites of $T$. gondii
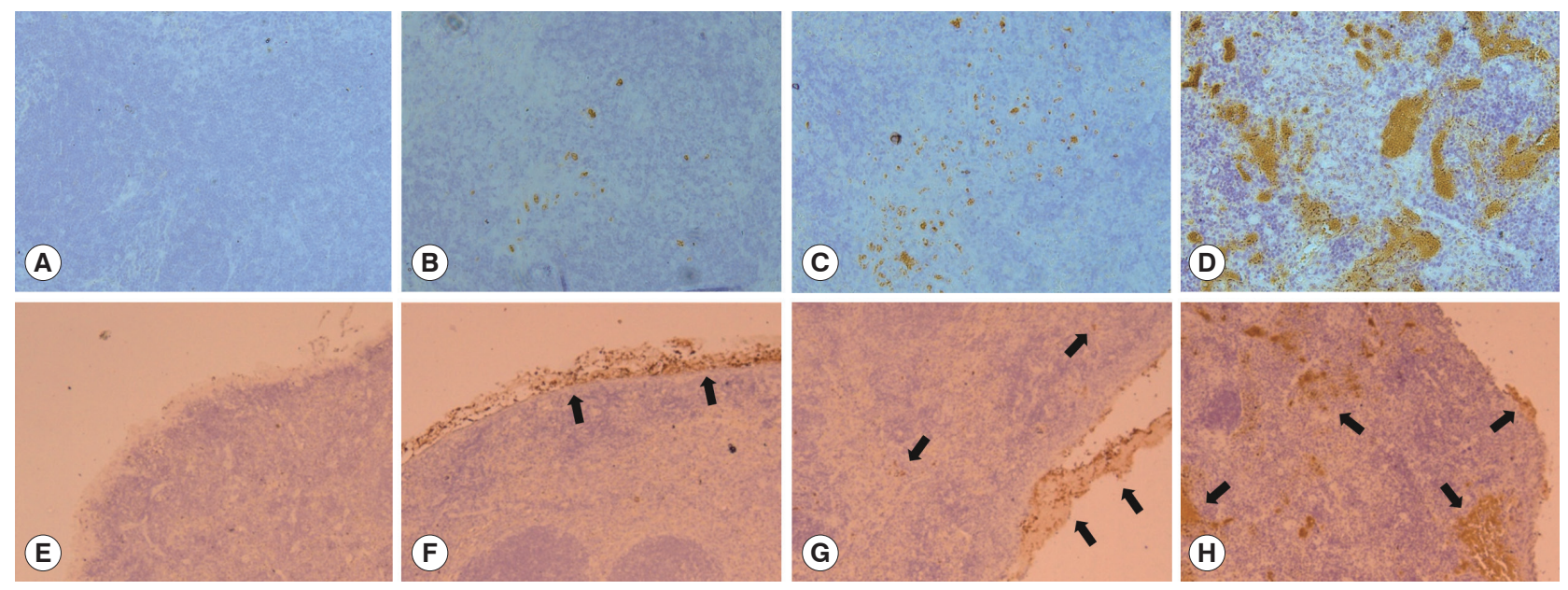

Fig. 2. Parasite infiltration detection by immunohistochemistry in spleen. Immunohistochemistry of the spleen section, showing T. gondii $\mathrm{RH}$ stained in brown. (A) There was no brown color in the spleen section of naive group. However, the other groups showed increased DAB reactions in an infection dose-dependent manner. (B) 10 tachyzoites infected mice. (C) 30 tachyzoites infected mice. (D) 100: tachyzoites infected mice. (E-H) T. gondii infiltration (arrow) in capsule area of spleen at day 9 post-infection. (E) The Naïve control mice, capsule tissue remained perfectly intact and there was no parasite invasion. $(F) 10$ tachyzoites infected mice showed capsule area infection. (G) 30 tachyzoites infected mice showed capsule and spleen tissue infection. $(H) 100$ tachyzoites infected mice showed infected capsule and several areas inside of the spleen. Photos are representative from 5 tissue sections. 
(RH) at day 9 post-infection. Ten tachyzoites infected mice showed lower parasite infiltration compared to 30 and 100 tachyzoites infected mice (Fig. 2B-D). One hundred tachyzoites infected mice showed the highest parasite infiltration as expected. As seen in Fig. 2, varying degrees of splenic capsule in mice infected by 10, 30 or 100 tachyzoites of T. gondii were found as well as T. gondii infectivity in the spleen. Splenic infiltration in 10 tachyzoites infected mice were mainly limited to splenic capsule area (Fig. 2F), whereas capsules from 30 tachyzoites infected mice appeared to be detached from the surface of spleens (Fig. 2G). Splenic capsule from 100 tachyzoites infected mice were completely detached and several infected areas of the spleen tissues were observed (Fig. 2H). These results indicated that low dose (10 tachyzoites infected mice) of infection induces $T$. gondii infection limited to the capsule area, whereas higher infection doses (30 and 100 tachyzoites infected mice) induce removal of T. gondii-infected capsules and enables infiltration of spleen tissues as well.

Mice were infected with 10, 30 or $100 \mathrm{~T}$. gondii (RH), and mouse survival was recorded daily. As seen in Fig. 3, 10 tachyzoites infected mice (10) survived significantly longer compared to 100 tachyzoites infected mice (100), although 100, 30, and 10 tachyzoites infected mice died at day 12, 15, and 17 post-infection, each respectively. These results indicated that 10 or 30 tachyzoites dosage could be used as challenge infection dosage in vaccine study, in which $\mathrm{T}$ or B cell immune responses could be detected at early stage of challenge infection.

In this study, we demonstrated that low dosage of $T$. gondii tachyzoite infection can cause tachyzoite infiltration and apoptosis at days 7 or 9 post-infection which has not been reported. We found that even very low dose of T. gondii tachyzo-

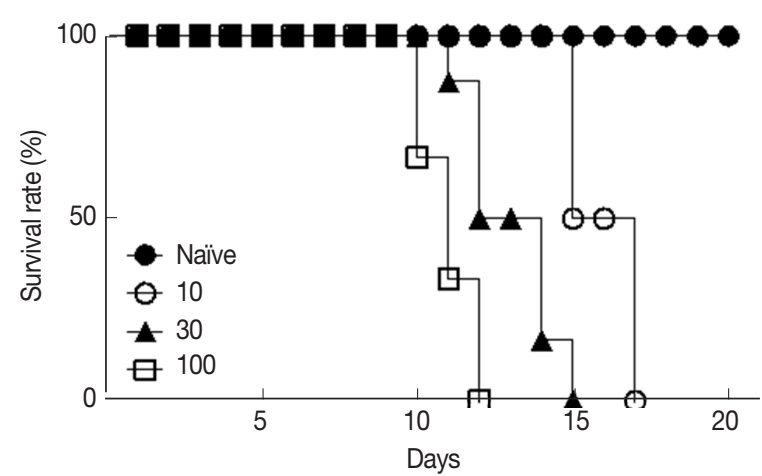

Fig. 3. Mouse survival. Mouse survival rate was monitored daily for 30 days after infections. Mice infected with 10, 30 or 100 tachyzoites. ites (10 tachyzoites) infection could induce parasite infiltration (infection) in the spleen and cause mouse death. Immunohistochemistry (IHC) is known to precisely label the location of the target antigen in cells and tissues [21]. In this study, we found tachyzoite infiltrations of $T$. gondii in the spleen at day 9 post-infection from 10, 30 and 100 tachyzoites doses by IHC. Higher dosage of infection (100 tachyzoites) caused higher levels of infiltration and apoptosis (Figs. 2, 3). As such, splenic infections were strictly limited to capsular areas of the spleen in 10 tachyzoites infected mice, while spleens of mice infected with higher doses were subjected to parasitic infiltration through the splenic capsule. Poor vaccine efficacies were observed from previous studies using high doses of tachyzoites for vaccine challenge experiment [5-12], indicating different vaccine efficacies might be concluded if low dosage of tachyzoites were used. Since all mice died from 10, 30, and 100 tachyzoites infections after spleen infiltration and apoptosis from 30 and 100 tachyzoites infected mice post-infection, we conclude that 30 tachyzoites dosage can be used for vaccine or apoptosis study.

Apoptosis can eliminate infected cells during virus, bacteria or parasite infections [17]. In the current study, low dose of tachyzoites (10 tachyzoites infected mice) infection was unable to induce apoptosis in spleen at day 9 post-infection whereas 30 or 100 tachyzoites infections induced significantly higher levels of apoptosis compared to naïve or 10 tachyzoites infection. However, since 10 tachyzoites infected mice died at day 17 post-infection, parasites infiltrated into the spleen and apoptosis in the spleen occurred and progressed after day 9. These results demonstrated that spleen is a very important organ that can be infected by tachyzoites of $T$. gondii, and parasitic infiltration and apoptosis induction can occur even with very low infection dose.

\section{ACKNOWLEDGMENTS}

This work was supported by grants from the National Research Foundation of Korea (NRF) (2018R1A2B6003535, 2018R1A6A1A03025124), the Cooperative Research Program for Agriculture Science \& Technology Development, Rural Development Administration, Republic of Korea (PJ01320501) and from Kyung Hee University in 2019 (KHU-20191220). 


\section{REFERENCES}

1. Montoya JG, Liesenfeld O. Toxoplasmosis. Lancet 2004; 363: 1965-1976.

2. Tenter AM, Heckeroth AR, Weiss LM. Toxoplasma gondii: from animals to humans. Int J Parasitol 2000; 30: 1217-1258.

3. Kim K, Weiss LM. Toxoplasma gondii: the model apicomplexan. Int J Parasitol 2004; 34: 423-432.

4. Moncada PA, Montoya JG. Toxoplasmosis in the fetus and newborn: an update on prevalence, diagnosis and treatment. Expert Rev Anti Infect Ther 2012; 10: 815-828.

5. Wang S, Wang Y, Sun X, Zhang Z, Liu T, Gadahi JA, Xu L, Yan R, Song $\mathrm{X}$, Li X. Protective immunity against acute toxoplasmosis in BALB/c mice induced by a DNA vaccine encoding Toxoplasma gondii $10 \mathrm{kDa}$ excretory-secretory antigen (TgESA10). Vet Parasitol 2015; 214: 40-48.

6. Wang S, Wang Y, Sun X, Zhang Z, Liu T, Gadahi JA, Hassan IA, $\mathrm{Xu}$ L, Yan R, Song X. Protective immunity against acute toxoplasmosis in BALB/C mice induced by a DNA vaccine encoding Toxoplasma gondii elongation factor 1-alpha. BMC Infect Dis 2015; 15: 448 .

7. Chen J, Huang SY, Zhou DH, Li ZY, Petersen E, Song HQ, Zhu XQ. DNA immunization with eukaryotic initiation factor- $2 \alpha$ of Toxoplasma gondii induces protective immunity against acute and chronic toxoplasmosis in mice. Vaccine 2013; 31: 6225-6231.

8. Guo J, Zhou A, Sun X, Sha W, Ai K, Pan G, Zhou C, Zhou H, Cong $\mathrm{H}, \mathrm{He}$ S. High immunogenicity of virus-like-particle vaccine containing multiple antigenic epitopes of Toxoplasma gondii against acute and chronic toxoplasmosis in mice. Front Immunol 2019; 10: 592.

9. Li ZY, Chen J, Petersen E, Zhou DH, Huang SY, Song HQ, Zhu XQ. Synergy of mIL-21 and mIL-15 in enhancing DNA vaccine efficacy against acute and chronic Toxoplasma gondii infection in mice. Vaccine 2014; 32: 3058-3065.

10. Lee SH, Kang HJ, Lee DH, Quan FS. Protective immunity induced by incorporating multiple antigenic proteins of Toxoplasma gondii into influenza virus-like particles. Front Immunol 2018; 9: 3073.

11. Lee SH, Kang HJ, Lee DH, Kang SM, Quan FS. Virus-like particle vaccines expressing Toxoplasma gondii rhoptry protein 18 and mi- croneme protein 8 provide enhanced protection. Vaccine 2018; 36: 5692-5700.

12. Lee SH, Kim AR, Lee DH, Rubino I, Choi HJ, Quan FS. Protection induced by virus-like particles containing Toxoplasma gondii microneme protein 8 against highly virulent RH strain of Toxoplasma gondii infection. PLoS One 2017; 12: e0175644.

13. Saraf P, Shwab EK, Dubey JP, Su C. On the determination of Toxoplasma gondii virulence in mice. Exp Parasitol 2017; 174: 2530.

14. Unno A, Kachi S, Batanova TA, Ohno T, Elhawary N, Kitoh K, Takashima Y. Toxoplasma gondii tachyzoite-infected peripheral blood mononuclear cells are enriched in mouse lungs and liver. Exp Parasitol 2013; 134: 160-164.

15. Montazeri M, Emami S, Asgarian-Omran H, Azizi S, Sharif M, Sarvi S, Rezaei F, Sadeghi M, Gohardehi S, Daryani A. In vitro and in vivo evaluation of kojic acid against Toxoplasma gondii in experimental models of acute toxoplasmosis. Exp Parasitol 2019; 200: 7-12.

16. Mordue DG, Monroy F, La Regina M, Dinarello CA, Sibley LD. Acute toxoplasmosis leads to lethal overproduction of Th1 cytokines. J Immunol 2001; 167: 4574-4584.

17. Elmore S. Apoptosis: a review of programmed cell death. Toxicol Pathol 2007; 35: 495-516.

18. Joyce BR, Queener SF, Wek RC, Sullivan WJ Jr. Phosphorylation of eukaryotic initiation factor-2 2 alpha\} promotes the extracellular survival of obligate intracellular parasite Toxoplasma gondii. Proc Natl Acad Sci USA 2010; 107: 17200-17205.

19. Chen J, Huang SY, Li ZY, Yuan ZG, Zhou DH, Petersen E, Zhang NZ, Zhu XQ. Protective immunity induced by a DNA vaccine expressing eIF4A of Toxoplasma gondii against acute toxoplasmosis in mice. Vaccine 2013; 31: 1734-1739.

20. Bastos da Silva I, Batista TP, Martines RB, Kanamura CT, Ferreira IM, Vidal JE, Pereira-Chioccola VL. Genotyping of Toxoplasma gondii: DNA extraction from formalin-fixed paraffin-embedded autopsy tissues from AIDS patients who died by severe disseminated toxoplasmosis. Exp Parasitol 2016; 165: 16-21.

21. Ramos-Vara JA, Miller MA. When tissue antigens and antibodies get along: revisiting the technical aspects of immunohistochemistry-the red, brown, and blue technique. Vet Pathol 2014; 51: 42-87. 
